\title{
Association between supratentorial pediatric high-grade gliomas involved with the subventricular zone and decreased survival: a multi-institutional retrospective study
}

\author{
Akshitkumar M. Mistry, MD, ${ }^{1}$ Nishit Mummareddy, BA, ${ }^{2}$ Travis S. CreveCoeur, BS, ${ }^{3}$ \\ Jock C. Lillard, MD, ${ }^{4}$ Brandy N. Vaughn, RN, ${ }^{4}$ Jean-Nicolas Gallant, PhD, ${ }^{5}$ Andrew T. Hale, PhD, ${ }^{5}$ \\ Natalie Griffin, MD, ${ }^{3}$ John C. Wellons III, MD, MSPH, ${ }^{1,6}$ David D. Limbrick Jr., MD, PhD, ${ }^{7}$ \\ Paul Klimo Jr., MD, MPH, ${ }^{4}$ and Robert P. Naftel, MD ${ }^{1,6}$

\begin{abstract}
'Department of Neurological Surgery, Vanderbilt University Medical Center; ${ }^{2}$ School of Medicine, Vanderbilt University, Nashville, Tennessee; ${ }^{3}$ School of Medicine, Washington University, St. Louis, Missouri; ${ }^{4}$ Department of Neurological Surgery, University of Tennessee Health Science Center, Memphis; ${ }^{5}$ Medical Scientist Training Program, School of Medicine, Vanderbilt University, Nashville; ${ }^{6}$ Vanderbilt Children's Hospital, Nashville, Tennessee; and 'Department of Neurosurgery, Washington University, St. Louis, Missouri
\end{abstract}

OBJECTIVE The subventricular zone (SVZ), housed in the lateral walls of the lateral ventricles, is the largest neurogenic niche in the brain. In adults, high-grade gliomas in contact or involved with the SVZ are associated with decreased survival. Whether this association holds true in the pediatric population remains unexplored. To address this gap in knowledge, the authors conducted this retrospective study in a pediatric population with high-grade gliomas treated at three comprehensive centers in the United States.

METHODS The authors retrospectively identified 63 patients, age $\leq 21$ years, with supratentorial WHO grade III-IV gliomas treated at three academic centers. Basic demographic and clinical data regarding presenting signs and symptoms and common treatment variables were obtained. Preoperative MRI studies were evaluated to assess SVZ contact by tumor and to quantify tumor volume.

RESULTS Sixty-three patients, including 34 males (54\%), had a median age of 12.3 years (IQR 6.50-16.2) and a median tumor volume of $39.4 \mathrm{ml}$ (IQR 19.4-65.8). Tumors contacting the SVZ (SVZ+) were noted in 34 patients (54\%) and overall were larger than those not in contact with the SVZ (SVZ-; 51.1 vs $27.3, p=0.002$ ). The SVZ+ tumors were also associated with decreased survival. However, age, tumor volume, tumor grade, and treatment with chemotherapy and/or radiation were not associated with survival in the 63 patients. In the univariable analysis, near-total resection, gross-total resection, and seizure presentation were associated with increased survival $(\mathrm{HR}=0.23,95 \% \mathrm{Cl} 0.06-0.88, p=0.03$; $\mathrm{HR}=0.26,95 \% \mathrm{Cl} 0.09-0.74, \mathrm{p}=0.01$; and $\mathrm{HR}=0.46,95 \% \mathrm{Cl} 0.22-0.97, \mathrm{p}=0.04$, respectively). In a multivariable stepwise Cox regression analysis, only SVZ+ tumors remained significantly associated with decreased survival $(\mathrm{HR}=$ $1.94,95 \% \mathrm{Cl} 1.03-3.64, \mathrm{p}=0.04)$.

CONCLUSIONS High-grade glioma contact with the SVZ neural stem cell niche was associated with a significant decrease in survival in the pediatric population, as it is in the adult population. This result suggests that tumor contact with the SVZ is a general negative prognosticator in high-grade glioma independent of age group and invites biological investigations to understand the SVZ's role in glioma pathobiology.

https://thejns.org/doi/abs/10.3171/2020.3.PEDS19593

KEYWORDS glioblastoma; glioma; subventricular zone; survival; pediatric brain tumors; oncology 
$\mathrm{T}$ HE subventricular zone (SVZ) is a growth factorrich niche in the brain that contains neural stem cells., ${ }^{1,2}$ Recently, many studies have shed light on the role the SVZ plays in gliomagenesis and in influencing glioma pathobiology. Pretreatment invasion of the SVZ niche by glioblastoma (GBM) independently leads to its increased malignancy, as evidenced by therapy resistance ${ }^{3}$ and significantly greater patient mortality. ${ }^{4-10}$ However, all of these observations have been made in adults. Whether invasion of the SVZ by high-grade gliomas (HGGs) prior to treatment is associated with lower survival in the pediatric population is unknown. Based on the results observed in adults, we hypothesized that HGG contact with the SVZ leads to poor pediatric outcomes, especially given that the SVZ is a much more active stem cell niche in children.11,12 To address this gap in knowledge, we conducted a retrospective study in a pediatric population with HGGs treated at three comprehensive centers in the United States.

\section{Methods}

\section{Patient Identification}

This study was approved by the institutional review boards of Vanderbilt University Medical Center (Nashville, TN), Washington University (St. Louis, MO), and Le Bonheur Children's Hospital (Memphis, TN). Patient consent was waived. All patients with an age $\leq 21$ years and a supratentorial, high-grade (WHO grade III or IV), diffuse glioma with the following histopathological diagnoses were identified by systematically querying institutional records: GBM, astrocytoma, oligodendroglioma, astroblastoma, and diffuse glioma not otherwise specified.

\section{Clinical Data Collection}

Patients' histopathological diagnosis, tumor grade, age at diagnosis, presentation symptomology, and treatment modalities were recorded. A radiologist along with a neurosurgeon assessed the extent of resection as biopsy, subtotal resection, near-total resection $(<5 \%$ residual), or gross-total resection based on the tumor-specific contrast enhancement remaining on postoperative MRI. For non- contrast-enhancing tumors, the postoperative tumor-specific T2/FLAIR hyperintensity was used to assess extent of resection. Patients were followed up until October 2017, at which point overall survivals were calculated. Study data were collected and managed using Research Electronic Data Capture (REDCap) electronic data capture tools hosted at Vanderbilt University Medical Center. ${ }^{13}$

\section{Radiological Data Collection}

All radiological assessments were performed using OsiriX Lite software (version 9.4, Pixmeo SARL) on axial MRI sequences. For clarifications, coronal or sagittal sequences (available or reformatted) were used. Preoperative MRI studies for all patients were analyzed to confirm supratentorial location, assess tumor invasion of or contact with the SVZ, and quantify tumor volume. Tumor invasion of or contact with the SVZ was designated according to an established method: ${ }^{6-8}$ contact or involvement of tumor mass enhancement with the lateral wall of the visible lateral ventricle - the location of the SVZ ${ }^{14}$ - on postgadolinium T1-weighted MRI. Figure 1 demonstrates examples of tumors with SVZ contact or involvement (SVZ+) and without (SVZ-). Tumors arising or located within the diencephalon were also noted. Tumor volume was quantified using the semi-automated volume-rendering function in OsiriX Lite after delineating the edges of the tumor mass. Assessments of both tumor contact with the SVZ and tumor volume segmentation were made independently by three experienced individuals (A.M.M., N.M., and R.P.N.) without knowledge of the patient outcomes. Any disagreement was reconciled by consensus review.

\section{Data Analysis}

Median and interquartile ranges were used to describe variable distributions. Medians were compared using a nonparametric Mann-Whitney U-test. Proportions of categorical variables were compared used Fisher's exact test. Survival time profiles were depicted using KaplanMeier plots. Overall survival time points were censored if patients were alive or their living status was unknown at the latest time of follow-up. Survival profiles were com-
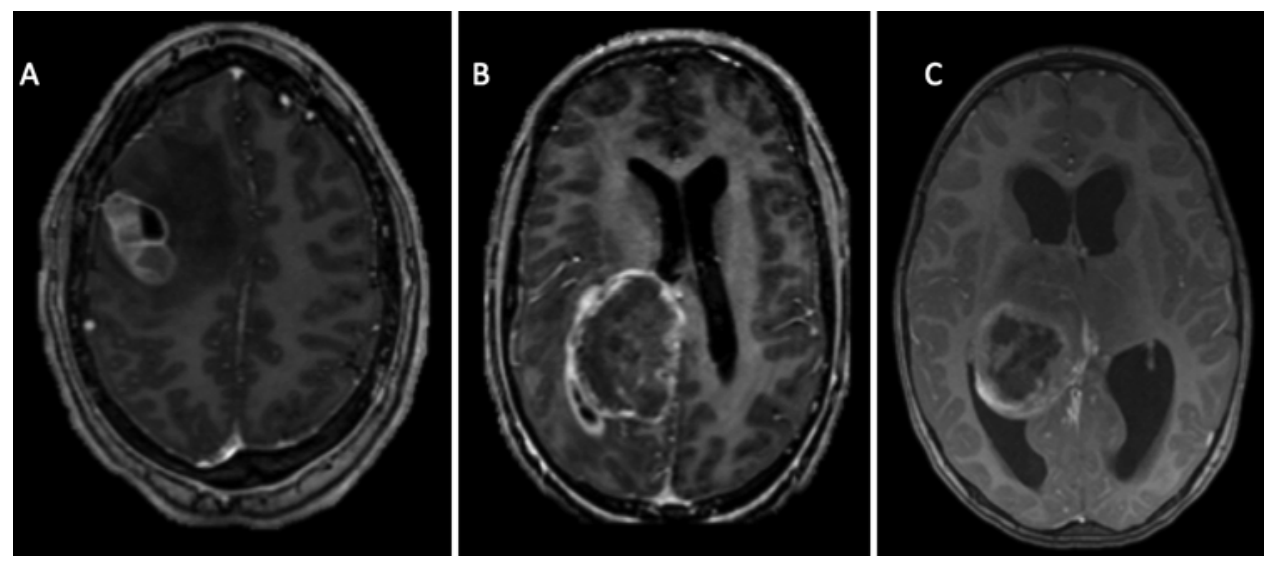

FIG. 1. Examples of preoperative axial MR images depicting HGG without (A) and with (B) enhancement involving the SVZ as well as a midline glioma (C) without SVZ contact. 

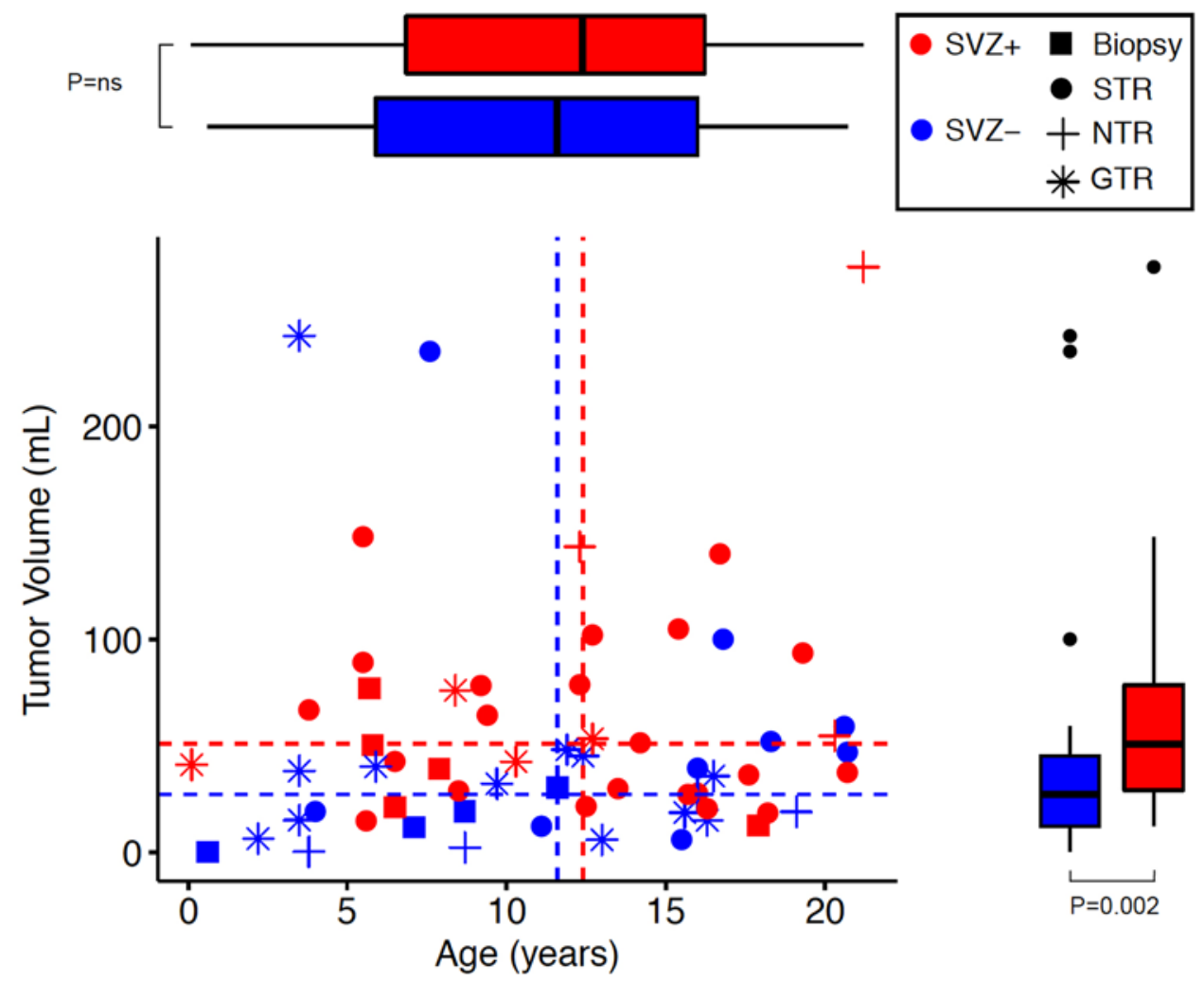

FIG. 2. Pediatric HGGs in contact with the SVZ were larger. Scatterplot depicts tumor volume, age, extent of resection, and SVZ tumor contact in 63 pediatric patients with HGG. Whether the tumor was (red) or was not (b/ue) in contact with the SVZ was based on preoperative MRI assessment. The box-and-whisker plots depict the distributions of age (upper) and tumor volume (right) based on SVZ contact. Thick vertical (upper) and horizontal (right) lines represent the medians of these distributions. GTR = gross-total resection; ns = not significant; NTR = near-total resection; STR = subtotal resection. Figure is available in color online only.

pared using a univariable log-rank (Mantel-Cox) test. To identify prognostic variables from a list of covariates, two stepwise Cox regression models were built computationally to ensure reliability: a conservative model with variable selection and exclusion criteria of $p<0.1$ and $>0.15$ and a stringent model with $\mathrm{p}<0.05$ and $>0.1$. To address any confounding effects, a Cox regression model was built including the variables associated with the primary variable. The results of these survival analyses are reported as hazard ratios with $95 \%$ confidence intervals. $\mathrm{R}$ version 3.5 (R Foundation for Statistical Computing) and MedCalc for Windows version 18.2 (MedCalc Software Ltd.) were used for all analyses and for making graphic plots. The survminer R package (version 0.4.3) was used to perform survival analyses, calculate median survival times, and plot right-censored Kaplan-Meier curves. Statistical significance, $\alpha$, was set at $\leq 0.05$ (two-sided) for all statistical analyses.

\section{Results}

\section{Overall Patient Characteristics}

We retrospectively identified 63 patients, whose median age was 12.3 years (IQR 6.5-16.2). Thirty-four patients (54\%) were males. All patients had been diagnosed between 2001 and 2016, except 2 patients who had been diagnosed in 1995 and 1998. Nearly half the patients had a GBM $(n=31)$. Others had been diagnosed with anaplastic astrocytoma (17 [27.0\%]), oligodendroglioma (9 [14.3\%]), diffuse glioma not otherwise specified (4 [6.3\%]), and astroblastoma (2 [3.2\%]). Sixteen patients (25.4\%) had undergone gross-total resection, $54(85.7 \%)$ had been treated with a chemotherapeutic agent, and 55 (87.3\%) had undergone postoperative radiation therapy. The median HGG volume was $39.4 \mathrm{ml}$ (IQR 19.4-65.8). Of note, there was no correlation between age and tumor volume (Pearson's correlation coefficient $0.06, p=0.65$; Fig. 2).

\section{Clinical and Radiographic Characteristics of SVZ+ and SVZ-}

Thirty-four patients (54\%) were found to have an SVZ+ HGG. The median age of these patients (12.4 vs 11.6 years, $p=0.57$; Table 1 and Fig. 2) and the frequencies of their queried presentation symptoms did not significantly differ from those in patients with SVZ- HGG. Tumor mutational status of $H 3 F 3 A$ (K27 or G34 mutations), BRAF, TP53, IDH1/2, MGMT, ATRX, and DAXX was available for 31 patients (Table 2). A majority of SVZ+ HGGs were diencephalic or midline gliomas (22/34 [64.7\%]). The median tumor volume of SVZ+ HGGs was significantly greater than that of SVZ- HGGs (51.1 vs $27.3 \mathrm{ml}, \mathrm{p}=0.002$; Table 
TABLE 1. Demographics and clinical characteristics of patients based on tumor contact with the SVZ

\begin{tabular}{|c|c|c|c|}
\hline Variable & SVZ- HGGs & SVZ+ HGGs & $\mathrm{p}$ Value \\
\hline No. of patients & 29 & 34 & \\
\hline Median age in yrs (IQR) & $11.6(5.90-16.0)$ & $12.4(6.85-16.2)$ & $0.57^{*}$ \\
\hline \multicolumn{4}{|l|}{ Presentation symptoms } \\
\hline Seizure & 11 & 8 & 0.27 \\
\hline Decreased mental status $\dagger$ & 3 & 8 & 0.20 \\
\hline Focal motor deficit & 4 & 7 & 0.53 \\
\hline Incidental & 2 & 0 & 0.21 \\
\hline Headaches & 14 & 21 & 0.32 \\
\hline Emesis & 11 & 12 & 0.99 \\
\hline Diencephalic/midline location & 4 & 22 & $<0.001$ \\
\hline Chemotherapy & 23 & 31 & 0.28 \\
\hline Radiation therapy & 25 & 30 & 0.99 \\
\hline Extent of resection & & & 0.02 \\
\hline Biopsy & 4 & 5 & \\
\hline Subtotal & 9 & 22 & \\
\hline Near total & 4 & 3 & \\
\hline Gross total & 12 & 4 & \\
\hline Histological diagnosis & & & 0.59 \\
\hline GBM & 12 & 19 & \\
\hline Anaplastic astrocytoma & 10 & 7 & \\
\hline Anaplastic oligodendroglioma & 5 & 4 & \\
\hline Anaplastic glioma, NOS & 1 & 3 & \\
\hline Astroblastoma & 1 & 1 & \\
\hline Median tumor vol in $\mathrm{ml}(\mathrm{IQR})$ & $27.3(12.4-45.4)$ & $51.1(29.3-78.8)$ & $0.002^{*}$ \\
\hline
\end{tabular}

1), and an overall lower extent of resection was achieved in SVZ+ HGGs $(\mathrm{p}=0.02)$.

\section{Prognosticators of Survival in Pediatric HGG}

Of the other variables collected in Table 1, univariable survival analysis revealed that extent of resection and seizure presentation were significantly associated with increased survival (Fig. 3A-C). Specifically, patients in whom near-total or gross-total resection had been achieved had a significantly longer survival profile than those who

TABLE 2. Tumor mutational status of select genes in 31 patients

\begin{tabular}{lcc}
\multicolumn{1}{c}{ Gene } & Patients w/ Known Status & No. w/ Mutation \\
\hline H3F3A & 5 & 0 \\
\hline BRAF & 16 & 2 \\
\hline TP53 & 13 & 4 \\
\hline IDH & 19 & 2 \\
\hline MGMT & 7 & 0 \\
\hline ATRX or DAXX & 8 & 3 \\
\hline
\end{tabular}

had undergone biopsy (univariable Cox HR $=0.23,95 \%$ CI $0.06-0.88, \mathrm{p}=0.03$; and HR $=0.26,95 \%$ CI $0.09-0.74$, $p=0.01$, respectively; $p=0.006$ for log-rank test of trend from biopsy to gross-total resection; Fig. 3B). Patients whose presenting symptom was seizure had a higher median survival than those without seizures at presentation (1012 vs 568 days, univariable Cox HR $=0.46,95 \%$ CI $0.22-0.97, p=0.04$; Fig. 3C). Of note, not age, tumor volume, tumor grade, nor midline tumor location correlated with survival. Additionally, treatment with chemotherapy and/or radiation was not associated with survival.

Patients with SVZ+ HGG had a lower median survival than those with SVZ- HGG (508 vs 981 days, univariable Cox HR $=1.94,95 \%$ CI 1.03-3.64, $\mathrm{p}=0.04$; Fig. 3A and D). In both the stringent and conservative multivariable models generated by stepwise selection of variables from all available variables, SVZ contact remained a prognosticator of survival in our study population. In building the stringent model, SVZ contact was the only variable computationally chosen that best accounted for the survival variability of the study patients. In the conservative model, seizure presentation $(\mathrm{HR}=0.47,95 \%$ CI $0.22-1.00, \mathrm{p}=$ $0.05)$ and tumor volume $(\mathrm{HR}=0.99,95 \% \mathrm{CI} 0.98-1.00, \mathrm{p}$ 
A

A
Age

Chemotherapy

Radiotherapy

Glioblastoma

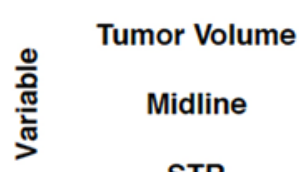

STR

NTR

GTR

SVZ Contact

Seizure Presentation

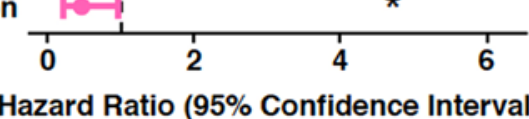

Hazard Ratio (95\% Confidence Interval)

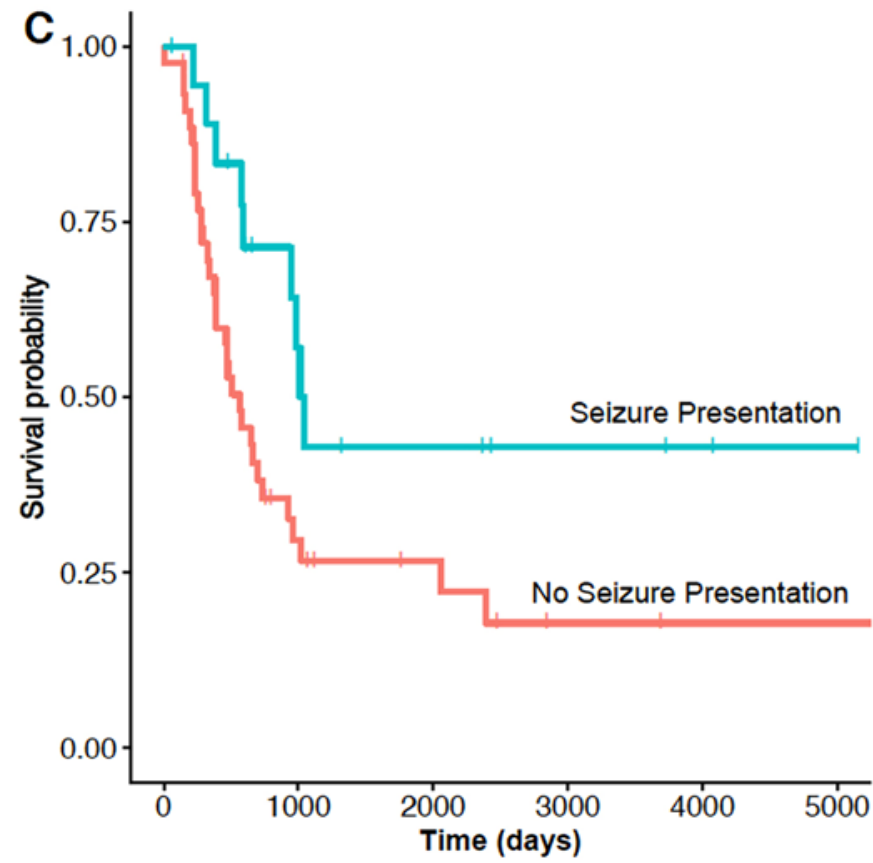

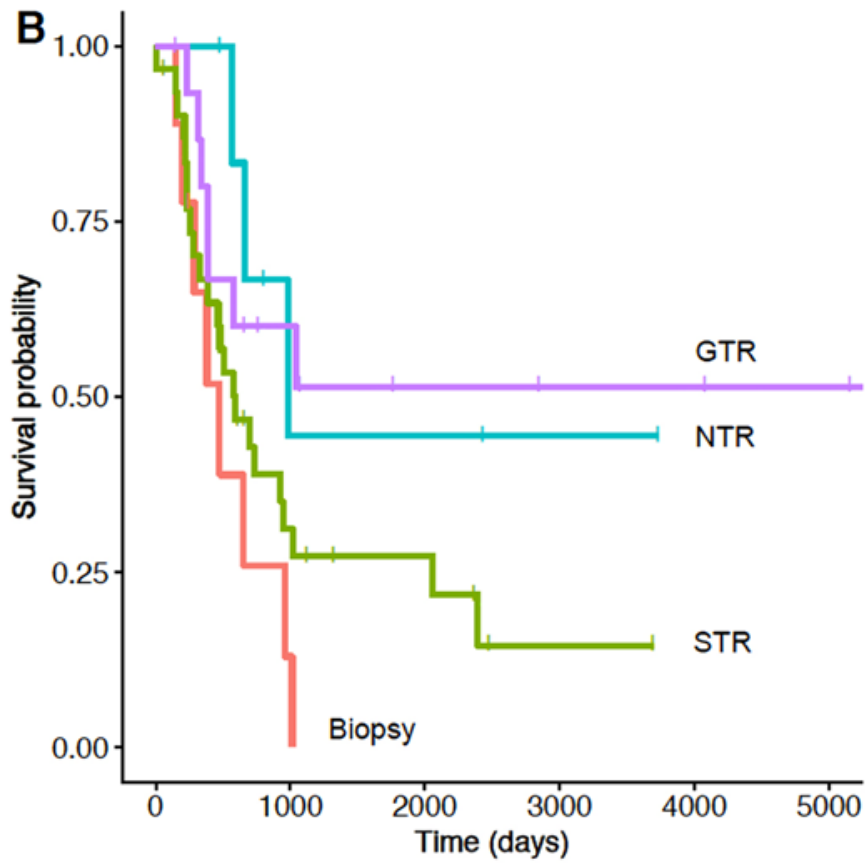

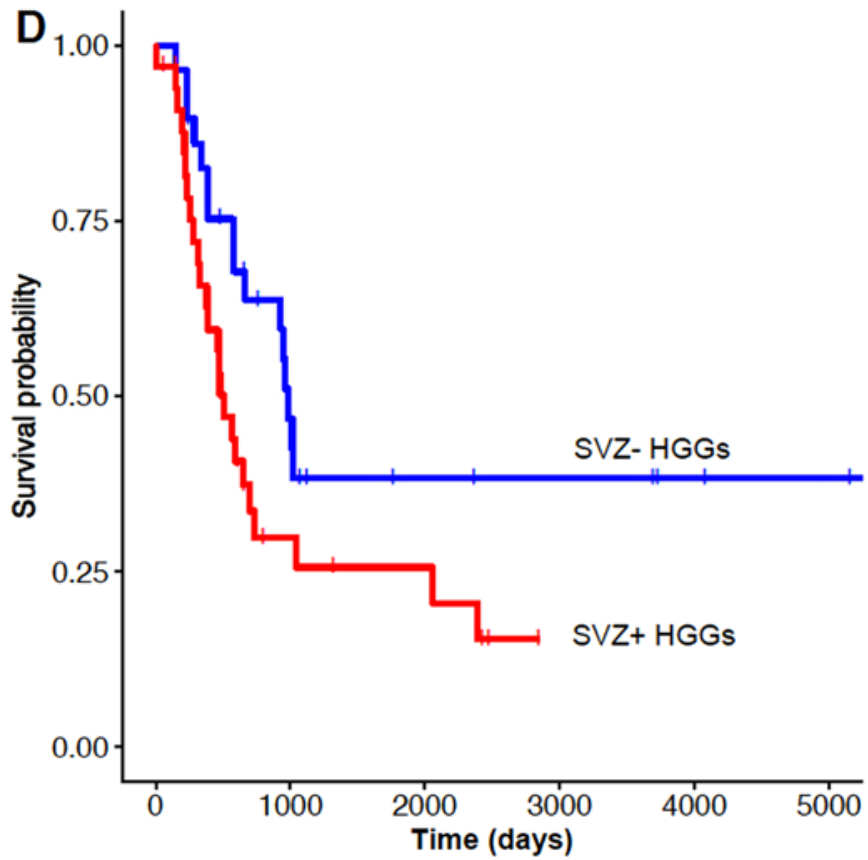

FIG. 3. Univariable survival analysis revealed that SVZ contact, NTR, GTR, and seizure presentation influence survival in pediatric HGG. The results of a univariable Cox analysis of all study variables compared to their respective negative controls (except for age and tumor volume, which are treated continuously) are represented in the forest plot $(\mathbf{A})$. The dot and the width of the line represent the hazard ratio and its associated $95 \%$ confidence interval, respectively. Asterisks identify a significant $(p \leq 0.05)$ result, which is depicted in a right-censored Kaplan-Meier survival curve (B-D). Censored values are identified with a vertical line on the curve. Figure is available in color online only.

$=0.08)$ were also selected in addition to SVZ contact $(\mathrm{HR}$ $=2.17,95 \%$ CI $1.10-4.28, \mathrm{p}=0.025)$. Lastly, the prognostic effect of SVZ contact was maintained in a Cox regression model manually adjusted for tumor volume and extent of resection (SVZ tumor contact $\mathrm{HR}=1.99,95 \% \mathrm{CI}$ $0.99-4.01, \mathrm{p}=0.05)$.

\section{Discussion}

To our knowledge, this is the first study demonstrating an association between SVZ contact by HGG and decreased survival in a pediatric population. Of the variables studied, SVZ contact or invasion by HGG was the only 
variable that best captured the survival variability of our study population. This result suggests that tumor contact with the SVZ is a general negative prognosticator in HGG independent of age group, as it is well demonstrated in adults with GBM, ${ }^{4-10}$ and invites biological investigations to understand the SVZ's role in glioma pathobiology.

Investigations are emerging that shed light on the mechanisms responsible for the increased malignancy of HGGs in contact with the SVZ in adults. For example, a recent clinical investigation has suggested that the increased mortality associated with SVZ+ HGGs may in part be attributable to an increased rate of tumor dissemination and hydrocephalus, which are independently associated with SVZ+ HGGs. ${ }^{15}$ The prior literature, summarized here, ${ }^{16}$ has suggested that ventricular entry, which occurs at a significantly higher rate in SVZ+ HGGs, ${ }^{15}$ may lead to increased tumor dissemination, hydrocephalus, or decreased survival. However, a head-to-head comparative analysis of ventricular entry and SVZ contact in adult GBM has indicated that SVZ contact, but not ventricular entry, drives these bad outcomes. ${ }^{15}$ This finding suggests that invasion of the SVZ, which has microarchitecture that facilitates cellular migration, ${ }^{17-19}$ by tumor from the parenchymal side also allows leptomeningeal spread of the tumor. It also increases direct access of neoplastic cells to CSF regardless of ventricular wall breach, increasing the probability of CSF tumor dissemination. The resulting increased protein/cellular CSF content can impair arachnoid granulations and lead to hydrocephalus. ${ }^{15,16,20}$ Complementing these clinical investigations, a basic investigation suggests that neoplastic cells resident in the SVZ are more resistant to radiation therapy under the influence of SVZspecific growth factors. ${ }^{3}$

In addition to confirming the above findings in the pediatric population, our results could lead to several investigations. For example, although no molecular difference was noted in bulk tumor analyses between adult SVZ+ GBM and SVZ- GBM ${ }^{8}$ it may be important to know whether pediatric SVZ+ HGGs are molecularly distinct from SVZ- HGGs, as nearly two-thirds of the SVZ+ HGGs in our study were diencephalic or midline gliomas, which are considered a molecularly distinct class of tumors with $\mathrm{K} 27 \mathrm{M}$ mutations in the histone $\mathrm{H} 3$ gene $H 3 F 3 A .{ }^{21}$ Thus, the limited availability of genetic data in our study is an important limitation, and our results need to be interpreted with this limitation in mind. Future studies need to investigate the molecular differences between SVZ+ and SVZ- tumors in children. Current evidence may imply that pediatric SVZ+ HGGs do not have a distinct intrinsic molecular profile from SVZ- HGGs, because all HGGs, including midline gliomas located even as distant as the pons, ${ }^{22}$ display migration toward the SVZ. ${ }^{23,24}$ SVZ contact is more likely to be a manifestation of this glioma tropism for the SVZ. In an effort to confirm this, we analyzed the molecular profiles of the 5 pediatric GBMs available in The Cancer Genome Atlas with corresponding MRI studies in The Cancer Imaging Archive. ${ }^{25}$ Of these 5, 3 were SVZ+ GBMs and their molecular profiles did not significantly differ from the 2 SVZ- GBMs. Of course, this analysis is significantly too underpowered to draw any meaningful conclusions and warrants a thorough investigation.
In our study population, presenting with seizure was correlated with greater survival on univariable analysis. This finding, although not the primary aim of this investigation, is in accordance with other reports on diffuse HGGs in adults ${ }^{26-28}$ and another report on the pediatric population. ${ }^{29}$ Although cortical involvement is presumed, the exact basis for this association is unclear. In our study population, presentation of seizure did not correlate with the SVZ status of the tumor (Table 1), nor with extent of resection ( $p=0.12$, Fisher's exact test), histology $(p=0.85$, Fisher's exact test), or tumor volume ( $p=0.14$, MannWhitney U-test). Dedicated studies are warranted to investigate the correlation of seizure presentation with survival.

Our clinical results are limited by the inherent bias associated with a retrospective study, lack of mutational data, and small sample size. These biases were limited by using a prespecified list of variables to collect and collecting data independent of the primary variable (SVZ contact) assessment. Although midline gliomas were defined anatomically because of the lack of mutational data given that many patients in our study were diagnosed before clinical testing of $H 3 F 3 A$ mutations was widely adopted, there is an excellent correlation between our anatomical designation and mutational status. ${ }^{30,31}$ Furthermore, assessment of SVZ contact was performed using the extent of tumor contrast enhancement on MRI. The reliability of the extent of contrast enhancement as a reflection of tumor location is unknown. We used this method to align the results of this study with the existing literature on SVZ contact. Lastly, our results must be confirmed in a larger sample size. Extent of resection and genetic tumor subtype may confound the association between SVZ contact and survival in our study sample size.

\section{Conclusions}

HGG contact or involvement with the SVZ neural stem cell niche was associated with decreased survival in our pediatric population of 63 patients, results similar to prior findings in the adult population. While these results must be confirmed with a larger sample size, they do suggest that tumor contact with the SVZ is a general negative prognosticator in HGG independent of age group and invite biological investigations to understand the SVZ's role in glioma pathobiology.

\section{Acknowledgments}

This publication was supported by Clinical and Translational Science Awards No. UL1 TR002243 from the National Center for Advancing Translational Sciences.

The contents of this publication are solely the responsibility of the authors and do not necessarily represent official views of the National Center for Advancing Translational Sciences of the National Institutes of Health or Microbot Medical Inc.

\section{References}

1. Sanai N, Tramontin AD, Quiñones-Hinojosa A, et al. Unique astrocyte ribbon in adult human brain contains neural stem cells but lacks chain migration. Nature. 2004;427(6976):740744.

2. Tong CK, Alvarez-Buylla A. SnapShot: adult neurogenesis in the V-SVZ. Neuron. 2014;81(1):220-220.e1. 
3. Goffart N, Lombard A, Lallemand F, et al. CXCL12 mediates glioblastoma resistance to radiotherapy in the subventricular zone. Neuro Oncol. 2017;19(1):66-77.

4. Jafri NF, Clarke JL, Weinberg V, et al. Relationship of glioblastoma multiforme to the subventricular zone is associated with survival. Neuro Oncol. 2013;15(1):91-96.

5. Liu S, Wang Y, Fan X, et al. Association of MRI-classified subventricular regions with survival outcomes in patients with anaplastic glioma. Clin Radiol. 2017;72(5):426.e1-426. e6.

6. Mistry AM, Dewan MC, White-Dzuro GA, et al. Decreased survival in glioblastomas is specific to contact with the ventricular-subventricular zone, not subgranular zone or corpus callosum. J Neurooncol. 2017;132(2):341-349.

7. Mistry AM, Hale AT, Chambless LB, et al. Influence of glioblastoma contact with the lateral ventricle on survival: a meta-analysis. J Neurooncol. 2017;131(1):125-133.

8. Mistry AM, Wooten DJ, Davis LT, et al. Ventricularsubventricular zone contact by glioblastoma is not associated with molecular signatures in bulk tumor data. Sci Rep. 2019;9(1):1842.

9. van Dijken BRJ, Jan van Laar P, Li C, et al. Ventricle contact is associated with lower survival and increased peritumoral perfusion in glioblastoma. J Neurosurg. 2018;131(3):717-723.

10. Young GS, Macklin EA, Setayesh K, et al. Longitudinal MRI evidence for decreased survival among periventricular glioblastoma. J Neurooncol. 2011;104(1):261-269.

11. Capilla-Gonzalez V, Herranz-Pérez V, García-Verdugo JM. The aged brain: genesis and fate of residual progenitor cells in the subventricular zone. Front Cell Neurosci. 2015;9:365.

12. Conover JC, Shook BA. Aging of the subventricular zone neural stem cell niche. Aging Dis. 2011;2(1):149-163.

13. Harris PA, Taylor R, Thielke R, et al. Research electronic data capture (REDCap) - a metadata-driven methodology and workflow process for providing translational research informatics support. J Biomed Inform. 2009;42(2):377-381.

14. Sanai N, Alvarez-Buylla A, Berger MS. Neural stem cells and the origin of gliomas. N Engl J Med. 2005;353(8):811-822.

15. Mistry AM, Kelly PD, Gallant JN, et al. Comparative analysis of subventricular zone glioblastoma contact and ventricular entry during resection in predicting dissemination, hydrocephalus, and survival. Neurosurgery. 2019;85(5):E924E932.

16. Mistry AM, Kelly PD, Thompson RC, Chambless LB. Cancer dissemination, hydrocephalus, and survival after cerebral ventricular entry during high-grade glioma surgery: a metaanalysis. Neurosurgery. 2018;83(6):1119-1127.

17. Gilbertson RJ, Rich JN. Making a tumour's bed: glioblastoma stem cells and the vascular niche. Nat Rev Cancer. 2007;7(10):733-736.

18. Hayashi Y, Nakada M, Tanaka S, et al. Implication of 5 -aminolevulinic acid fluorescence of the ventricular wall for postoperative communicating hydrocephalus associated with cerebrospinal fluid dissemination in patients with glioblastoma multiforme: a report of 7 cases. J Neurosurg. 2010;112(5):1015-1019.

19. Iacoangeli M, Di Rienzo A, Colasanti R, et al. Endoscopyverified occult subependymal dissemination of glioblastoma and brain metastasis undetected by MRI: prognostic significance. OncoTargets Ther. 2012;5:449-456.

20. Krishnamurthy S, Li J. New concepts in the pathogenesis of hydrocephalus. Transl Pediatr. 2014;3(3):185-194.

21. Louis DN, Perry A, Reifenberger G, et al. The 2016 World Health Organization Classification of Tumors of the Central Nervous System: a summary. Acta Neuropathol. 2016;131(6):803-820.

22. Caretti V, Bugiani M, Freret M, et al. Subventricular spread of diffuse intrinsic pontine glioma. Acta Neuropathol. 2014;128(4):605-607.
23. Goffart N, Kroonen J, Di Valentin E, et al. Adult mouse subventricular zones stimulate glioblastoma stem cells specific invasion through CXCL12/CXCR4 signaling. Neuro Oncol. 2015;17(1):81-94.

24. Qin EY, Cooper DD, Abbott KL, et al. Neural precursorderived pleiotrophin mediates subventricular zone invasion by glioma. Cell. 2017;170(5):845-859.e19.

25. Clark K, Vendt B, Smith K, et al. The Cancer Imaging Archive (TCIA): maintaining and operating a public information repository. J Digit Imaging. 2013;26(6):1045-1057.

26. Fan X, Li Y, Shan X, et al. Seizures at presentation are correlated with better survival outcomes in adult diffuse glioma: a systematic review and meta-analysis. Seizure. 2018;59:16-23.

27. Flanigan PM, Jahangiri A, Kuang R, et al. Improved survival with decreased wait time to surgery in glioblastoma patients presenting with seizure. Neurosurgery. 2017;81(5):824-833.

28. Lu VM, Jue TR, Phan K, McDonald KL. Quantifying the prognostic significance in glioblastoma of seizure history at initial presentation: a systematic review and meta-analysis. Clin Neurol Neurosurg. 2018;164:75-80.

29. Walston S, Hamstra DA, Oh K, et al. A multi-institutional experience in pediatric high-grade glioma. Front Oncol. 2015;5:28.

30. Sturm D, Witt H, Hovestadt V, et al. Hotspot mutations in H3F3A and IDH1 define distinct epigenetic and biological subgroups of glioblastoma. Cancer Cell. 2012;22(4):425-437.

31. Wang L, Li Z, Zhang M, et al. H3 K27M-mutant diffuse midline gliomas in different anatomical locations. Hum Pathol. 2018;78:89-96.

\section{Disclosures}

Dr. Limbrick receives research funding for unrelated projects from Microbot Medical Inc. and Medtronic Inc.

\section{Author Contributions}

Conception and design: Mistry, Wellons, Limbrick, Klimo, Naftel. Acquisition of data: Mistry, Mummareddy, CreveCoeur, Lillard, Vaughn, Gallant, Hale, Griffin. Analysis and interpretation of data: Mistry, Mummareddy, Lillard, Gallant, Hale, Griffin, Wellons, Limbrick, Klimo, Naftel. Drafting the article:

Mistry, Mummareddy. Critically revising the article: Mistry, Mummareddy, Wellons, Limbrick, Klimo, Naftel. Reviewed submitted version of manuscript: Mistry, Mummareddy, CreveCoeur, Lillard, Vaughn, Gallant, Hale, Griffin, Wellons, Limbrick, Naftel. Approved the final version of the manuscript on behalf of all authors: Mistry. Statistical analysis: Mummareddy. Study supervision: Mistry, Wellons, Limbrick, Klimo, Naftel.

\section{Supplemental Information}

\section{Previous Presentations}

The results of this study were presented orally at the 47th Annual Meeting of the AANS/CNS Section on Pediatric Neurological Surgery, December 6-9, 2018, in Nashville, TN.

\section{Correspondence}

Akshitkumar M. Mistry: Vanderbilt University Medical Center, Nashville, TN. axitamm@gmail.com. 